\title{
A WIRELESS FUNCTIONAL ELECTRICAL STIMULATION SYSTEM
}

\author{
Adrian Derungs ${ }^{1}$, Christian Dietrich ${ }^{1}$ and Kenneth J. Hunt ${ }^{1}$ \\ ${ }^{1}$ Institute for Rehabilitation and Performance Technology \\ Division of Mechanical Engineering, Department of Engineering and Information Technology \\ Bern University of Applied Sciences, Switzerland \\ adrian.derungs@bfh.ch
}

\begin{abstract}
A system for wireless electrical stimulation was designed and the feasibility evaluated. The Bluetooth communication standard and two different microcontroller systems provided the basis of the system design. The prototype allows four channels of stimulation whereby each stimulator unit contained two channels. The design permitted the possibility of generating voltage controlled biphasic stimulation patterns for muscle activation. The accuracy of stimulation parameters including pulse width, inter-pulse time, baud rate, latency, range, stimulator power consumption and also muscle stimulation were evaluated and feasiblity thereby established. Further research should solve the problem of latency in wireless communication and extend the system with sensors to design closed-loop control strategies.
\end{abstract}

Keywords: wireless, Bluetooth, Real-Time Operating System, four-channel FES, applications

\section{Introduction}

Functional Electrical Stimulation (FES) provides various approaches for rehabilitation of patients who suffer paraplegia or stroke. FES allows restoring and maintaining motion, and activities of daily living (ADL) can be performed. This gives disabled people the opportunity for independence. These facts served as the motivation to implement a novel wireless device for FES. The aim of this research project was the development of a complete system to demonstrate the feasibility of wireless stimulation with four channels. A key design requirement was that each remote stimulation unit had two channels, purposely allowing activation locally of a pair of agonist-antagonist muscle groups at a given joint. The scope was defined by the requirement to evaluate feasibility. The literature shows one provisional paper regarding a wireless FES solution [1]. Most commercially available products for FES in sport and rehabilitation are not currently wireless (an exception is the COMPEX Wireless system from Compex) but the technology is promising for a range of products and applications.

\section{Methods}

The wireless FES system consists of two stimulators each with two channels to stimulate an antagonistic pair of muscles (Fig. 1) and one coordinator controlling the stimulators. The basis for the coordinator was a microcontroller evaluation board (MCBSTM32EXL, Keil). The coordina-

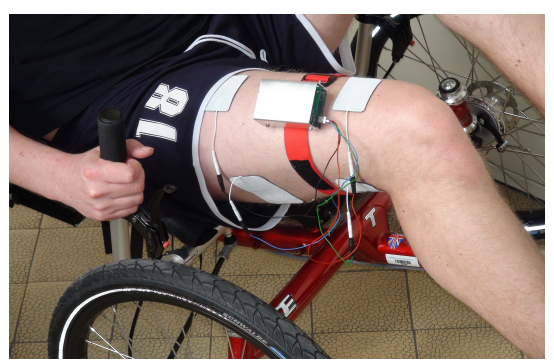

Figure 1: One stimulator with two channels.

tor ran with a real-time operating system (Keil RTX) which allowed adjustment of stimulation patterns and Bluetooth configuration via a joystick. For data transmission, Bluetooth modules were connected to the microcontrollers. For mobility, the stimulator was supplied by a $7.4 \mathrm{~V} \mathrm{Li}$-Po battery and enclosed in a $70 \times 52 \times 35 \mathrm{~mm}$ portable case fitted with a belt to mount the stimulator to the limbs. The stimulator was voltage regulated and generated biphasic pulses of $30 \mathrm{~V}$ or $50 \mathrm{~V}$ using a boost converter. An 8-Bit microcontroller (Atmega328 Mini R5, Arduino) was used to control the stimulation patterns. The code was written with Arduino's integrated development environment (IDE). To display communication and stimulation status, LEDs were mounted on the board. For muscle stimulation, surface electrodes were used, connected to the board via USB connectors. The stimulator algorithm interpreted received data and generated desired pulses. Several modes were programmed to select the number of activated channels and pulse patterns. The pattern (Fig. 2), was defined by parameters Mode, PulseTime, InterPulseTime, RestOfPeriod, Repetitions, Drift and OverallRepetitions, thus determining a desired stimulation frequency.

\section{Results}

A prototype for wireless stimulation with four channels was developed. The use of Bluetooth modules enabled a communication range of $45 \mathrm{~m}$ indoors. Boost converter output voltage was reliable and stable and had a deviation to the calculated output voltage of $2.2 \%$ for the stimulator with $30 \mathrm{~V}$ and $1.2 \%$ for the stimulator with $50 \mathrm{~V}$. Modulation time of signals with respect to baud rate was calculated to be maximally $9.8 \%$ with respect to measured modulation time. Therefore measurements with different amounts of data were made.

Additionally, it was demonstrated that latency for data 


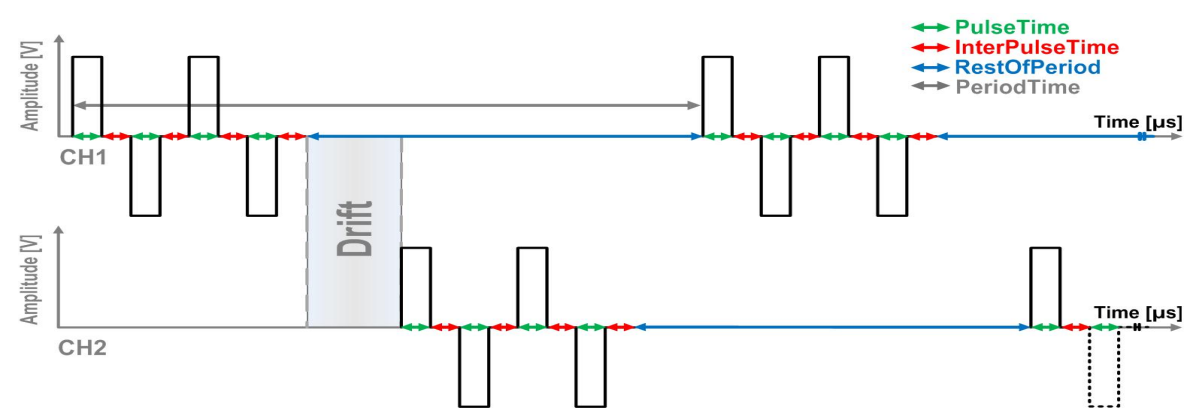

Figure 2: Two channel stimulation patterns with drift-time, the delay between antagonistic muscles.

transmission was $16.82 \mathrm{~ms}, 20.57 \mathrm{~ms}$ and $41.76 \mathrm{~ms}$ for baud rates of 115200 Baud, 57600 Baud, and 9600 Baud, respectively. Within this measurement series $(n=20)$ the amount of data remained constant (190 Bits). A second measurement concerning latency used the same baud rate $(115200$ bits per second) but varied the amount of data (10 Bits to 54000 Bits averaged over a series of 10 measurements). This showed a trend towards increased latency with increasing amounts of data.

Stimulation was as expected for different loads. For a pure resistive load the current followed the specified biphasic rectangular shape. For loads with capacitive elements (tissue and muscle) the current had an exponentially decreasing shape. Considering adjusted pattern parameters, $20 \%$ of the parameters tested deviated more than the specified $10 \%$. Fig. 3 shows an acceptable pulse width deviation. Performance of the new voltage-driven muscle stimulator was further evaluated by comparing its waveform with that of a commercial current-controlled stimulator (RehaStim, Hasomed GmbH, Germany). Typical behavioural characteristics are shown in Fig. 4.

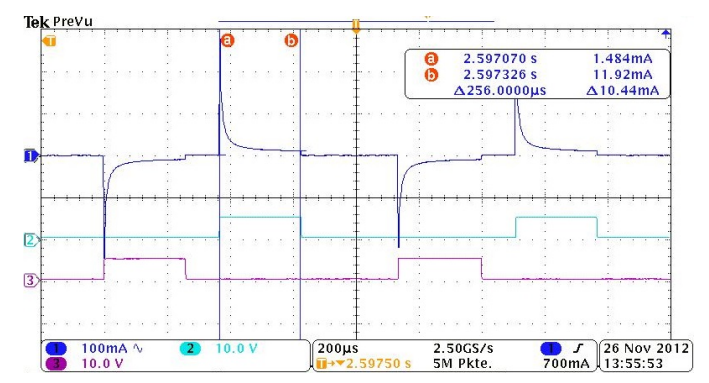

Figure 3: Measured $(256 \mu \mathrm{s})$ vs. specified $(250 \mu \mathrm{s})$ pulse width.

\section{Discussion}

The objective of a mobile stimulation unit with four channels, i.e. two pairs of two channels, was fulfilled. The measured output voltage of the booster corresponded with specifications given in the datasheet. The required time for data modulation was determined analytically and coincided with the measurements. Handling Bluetooth latency is a challenge for further development as a consequence of its inconsistency. It was observed that latency correlated with baud rate and with the amount of data. Further research in the details of the Bluetooth standard may allow optimisation of latency problems.

We conclude that the successful implementation and evaluation of a wireless FES system with four channels was technically feasible. The decision to implement a system based on a microcontroller was justified by the demand for mobility. Despite the limitations of Bluetooth, the presented work has potential for further technical development towards effective wireless stimulation solutions.

Future research should address the challenges of wireless communication including latency, where low-power radio technology (e.g. ANT+) could be an alternative approach. Furthermore the number of channels must be increased for an application like FES-cycling [2]. Additional topics for further development include pulse pattern waveforms, variable frequency trains and advanced wireless-based and local closed-loop control of induced muscle contractions [3], thus aiming towards more physiological stimulation to overcome rapid muscle fatigue.

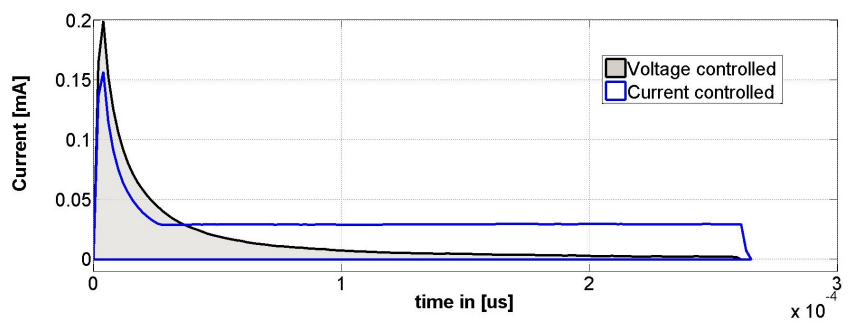

Figure 4: Voltage- vs. current-controlled muscle stimulation.

\section{Bibliography}

[1] N. Jovicic, L. Saranovac, and D. Popovic, "Wireless distributed functional electrical stimulation system," Journal of NeuroEngineering and Rehabilitation, vol. 9, no. 1, p. 54, 2012.

[2] K. J. Hunt, J. Fang, J. Saengsuwan, M. Grob, and M. Laubacher, "On the efficiency of FES cycling: A framework and systematic review," Technology and Health Care, vol. 20, no. 5, pp. 395-422, 2012.

[3] C. Lynch and M. Popovic, "Functional electrical stimulation," IEEE Control Systems Magazine, vol. 28, pp. $40-50$, April 2008. 